\title{
Einfache Kalibrierung von koplanaren Tastköpfen zur Messung der komplexen Permittivität
}

\author{
T. Reinecke, S. Ahrens, S. Zimmermann \\ Institut für Grundlagen der Elektrotechnik und Messtechnik, Fachgebiet Sensorik und Messtechnik, \\ Leibniz Universität Hannover, Hannover, Deutschland
}

\begin{abstract}
Kurzfassung
In vorangegangen Arbeiten wurde die Entwicklung eines koplanaren Tastkopfs für dielektrische Messungen beschrieben. Dazu wird der Tastkopf auf die zu charakterisierende Probe gepresst und die Transmission einer elektromagnetischen Welle mit einem Netzwerkanalysator gemessen. Aus dieser Messung kann die sogenannte effektive Permittivität $\varepsilon_{e f f}$ berechnet werden. Da sich bei einer Koplanarleitung die elektromagnetische Welle gleichzeitig in der Probe und in der Leiterplatte ausbreitet, ergibt sich $\varepsilon_{e f f}$ aus einer Überlagerung der relativen Permittivität der Probe $\varepsilon_{\text {Probe }}$ und der relativen Permittivität der Leiterplatte $\varepsilon_{\text {Substrat }}$. Um aus der effektiven Permittivität $\varepsilon_{\text {eff }}$ die relative Permittivität der Probe $\varepsilon_{\text {Probe }}$ zu bestimmen, muss der Tastkopf kalibriert werden. Hierzu wird in dieser Arbeit ein bekannter Kalibrieralgorithmus an den verwendeten Tastkopf angepasst, sodass die Kalibrierung mittels Messung von zwei Materialien bekannter Permittivität durchgeführt werden kann. Die Verifikation der Kalibrierung erfolgt anhand der Messung von Wasser-Ethanol-Mischungen und Vergleich der gemessenen relativen Permittivität mit der theoretisch berechneten Permittivität.
\end{abstract}

Keywords: Koplanarer Tastkopf, effektive Permittivität, Kalibrierverfahren, dielektrische Spektroskopie

\section{Einleitung}

Die Charakterisierung von Materialien anhand ihrer dielektrischen Eigenschaften wird in vielen Bereichen angewandt. Neben einer großen Zahl industrieller Anwendungen [1] kann zum Beispiel die Qualität von Fleischprodukten [2] oder der der Fettanteil in Fisch [3] beurteilt werden. Weiterhin werden dielektrische Messungen in einer Vielzahl medizinischer Anwendungen genutzt, zum Beispiel zur Bestimmung der Energieabsorption und möglicher Schadwirkung elektromagnetischer Wellen im menschlichen Gehirn, Bestimmung von Brustkrebs oder Charakterisierung von Gewebe [4-6]. Für die Messung der dielektrischen Eigenschaften, beziehungsweise der Permittivität einer Probe existieren diverse Ansätze [7]. Eine Möglichkeit besteht in der Verwendung von Streifenleitungen. Diese haben den Vorteil einer vergleichsweise einfachen Kalibrierung und ermöglichen sowohl Transmissions- als auch Reflexionsmessungen. Weiterhin können sie als Tastkopf ausgeführt werden, wodurch eine Probenpräparation entfällt und schnelle zerstörungsfreie Messungen ermöglicht werden. In [8] ist der Tastkopf aus Abb. 1 beschrieben. Dieser wurde für die quantitative Bestimmung des Wassergehalts in parenchymatösem Gewebe im Rahmen einer gerichtsmedizinischen Untersuchung entwickelt. Darauf aufbauend konnte dieser aber auch erfolgreich in anderen Anwendungen genutzt werden, wie zum Beispiel zur Überwachung des Zellwachstums in Bioreaktoren [9]. Der Tastkopf besteht aus einem Polyoxymethylen (POM) Substrat auf dem ein Innenleiter zentriert zwischen zwei Masseflächen aufgebracht ist.

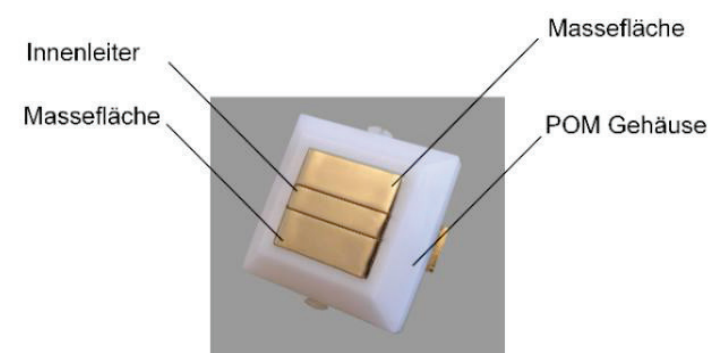

Abb. 1: Koplanarer Tastkopf

Um eine definierte Kontaktfläche zu erhalten, ist die Messfläche von einem POM Gehäuse umschlossen, unter dem die Leiterbahnen zu SMA-Kontakten weitergeführt werden. Um die Permittivität einer Probe zu messen, wird der Tastkopf auf die Probe gepresst und mittels 
Netzwerkanalysator die Transmission einer elektromagnetischen Welle durch den Tastkopf gemessen. Diese Transmission wird als Verhältnis zwischen hinlaufender Welle $a_{l}$ und transmittierter Welle $b_{2}$ beschrieben durch den Streuparameter

$$
S_{21}=\frac{b_{2}}{a_{1}}=\frac{z \cdot\left(1-\Gamma^{2}\right)}{1-z^{2} \Gamma^{2}} .
$$

In [10] ist der mathematische Zusammenhang zwischen $S_{21}$ und dem Reflexionsfaktor $\Gamma$ und dem Transmissionskoeffizienten $z$ gegeben. In Abb. 2 sind ein Querschnitt des Tastkopfes und des elektrischen Felds einer elektromagnetischen Welle dargestellt. Es ist zu erkennen, dass sich das Feld der Welle sowohl innerhalb der Probe als auch innerhalb der Leiterbahn ausbreitet.

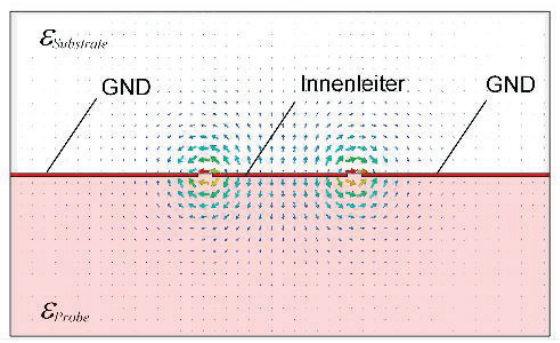

Abb. 2: Elektrisches Feld im koplanaren Tastkopf

Damit stellt die Koplanarleitung einen Spezialfall dar, da die Wellenausbreitung durch die effektive Permittivität $\varepsilon_{\text {eff. }}$, bestimmt wird. Diese ergibt sich aus einer Überlagerung der relativen Permittivität der Probe $\varepsilon_{\text {Probe }}$ und der relativen Permittivität der Leiterplatte $\varepsilon_{\text {Substrat }}$. Da $\Gamma$ und $z$ jeweils abhängig von der effektiven Permittivität sind, kann diese nach [10] nummerisch aus (1) bestimmt werden. Weitere Ausführungen zu den Besonderheiten bei der Bestimmung der effektiven Permittivität bei Verwendung des beschriebenen Tastkopfs finden sich in [8]. Im Allgemeinen ist der Realteil einer Permittivität ein Maß für die Polarisierbarkeit des Materials und der Imaginärteil ein $\mathrm{Ma} ß$ für die dielektrischen Verluste. Für die effektive Permitivität $\varepsilon_{e f f}$ hingegen, gelten diese Zusammenhänge nicht, siehe Abb. 2. Hier ist anhand einer CST Microwave Studio 2014 Simulation der Realteil der effektiven Permittivität in Abhängigkeit des Imaginärteils der Permittivität der Probe dargestellt, wobei der Realteil der Probenpermittivität konstant gehalten wird. Es ist zu erkennen, dass eine Erhöhung der dielektrischen Verluste in der Probe zu einem erhöhten Realteil der effektiven Permittivität führt. Messtechnisch kann dieser Effekt anhand von Messungen verschiedener Salzkonzentrationen in Wasser bestätigt werden.

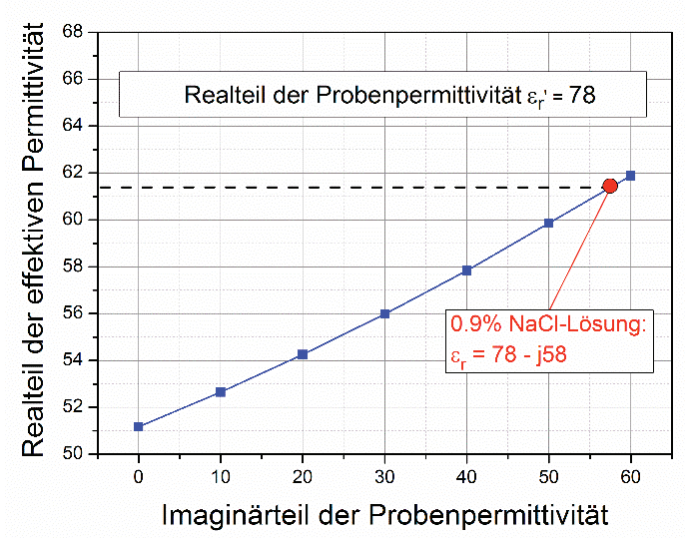

Abb. 3: CST Simulation: Imaginärteil der Probenpermittivität beeinflusst den Realteil der effektiven Permittivität.

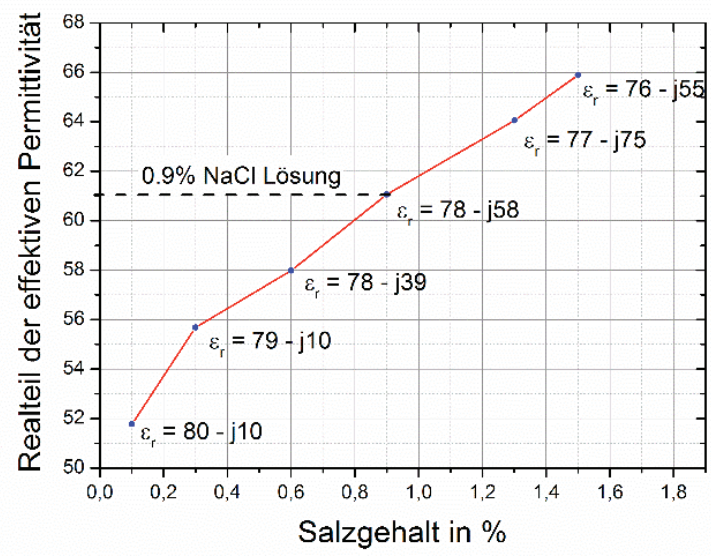

Abb. 4: Bestimmung der effektiven Permittivität von Salzlösungen verschiedener Konzentration

In Abb. 5 ist der Realteil der effektiven Permittivität in Abhängigkeit der Salzkonzentration in der Lösung dargestellt. An die jeweiligen Messpunkte ist die nach [11] theoretisch berechnete relative Permittivität der Salzlösung bei $20{ }^{\circ} \mathrm{C}$ angetragen. Es ist eine sehr gute Übereinstimmung zu den Simulationsergebnissen aus Abb. 3 zu erkennen. Obwohl der Realteil der Permittivität der Salzlösung sinkt, steigt der Realteil der effektiven Permittivität, bedingt durch den zunehmenden Imaginärteil der Permittivität der Salzlösung. Werden Proben vermessen, bei denen sich Polarisierbarkeit, dielektrische Verluste und Leitfähigkeit unabhängig voneinander verändern, führt der beschriebene Effekt dazu, dass die effektive Permittivität ihre Aussagekraft einbüßt und somit keinerlei Rückschlüsse auf die Permittivität der Probe möglich sind. In dieser Arbeit wird daher ein einfacher Ansatz zur Kalibrierung des koplanaren Tastkopfes verwendet, womit eine Berechnung der Permittivität der Probe aus der ermittelten effektiven Permittivität möglich ist. 


\section{Kalibrierung des koplanaren Tastkopfes}

In [12] wird der Zusammenhang zwischen effektiver Permittivität und der Permittivität der Probe beschrieben als

$$
\varepsilon_{\text {eff }}=\mathrm{m} \varepsilon_{\text {Probe }}+c .
$$

Hierbei ist zu beachten, dass alle Größen in dieser Gleichung komplexwertig sind. Speziell anhand der Steigung $m$ lässt sich ein steigender Realteil der effektiven Permittivität bei steigendem Imaginärteil der Permittivität der Probe erklären wie in Abb. 3 und 4 gezeigt. Aus (2) ergibt sich, dass $m$ und $c$ anhand von Messung zweier Materialien mit bekanntem Dielektrikum bestimmt werden können zu

$$
m=\frac{\varepsilon_{\text {eff }, 1}-\varepsilon_{\text {eff }, 2}}{\varepsilon_{\text {Probe }, 1}-\varepsilon_{\text {Probe }, 2}}
$$

und

$$
c=\varepsilon_{e f f, 1}-m \varepsilon_{\text {Probe }, 1} \text {. }
$$

In [12] werden dazu Luft und DI-Wasser verwendet. Zunächst wird der Tastkopf ebenfalls mit diesen beiden Materialien kalibriert. Alle Messungen werden bei einer Frequenz von $\mathrm{f}=500 \mathrm{MHz}$ durchgeführt. Hier hat Luft mit einer relativen Permittivität von $\varepsilon_{\text {Luft }}=1$ und DI-Wasser eine relative Permittivität (bei $25^{\circ} \mathrm{C}$ ) von $\varepsilon_{\text {Wasser }}=78-j 2$. Abweichend von dem Verfahren aus [12] bei dem die Ausbreitungskonstante der elektromagnetischen Welle aus Messungen an zwei unterschiedlich langen Koplanarleitungen ermittelt wird, wird in dieser Arbeit, wie in [8] beschrieben, direkt die effektive Permittivität bestimmt. Dazu wird zunächst der Netzwerkanalysator mittels $\mathrm{T}$ (rough) $\mathrm{O}$ (pen) $S$ (hort) $M$ (atch) Kalibrierung kalibriert. Da der koplanare Tastkopf an Luft auf eine Impedanz von $50 \Omega$ ausgelegt ist treten für diesen Fall keine Reflexionen auf und der Reflektionsfaktor hat somit den Wert $\Gamma=0$. Damit vereinfacht sich Gleichung (1) zu

$$
S_{21}=z=e^{-j \cdot \frac{\omega}{c_{0}} \cdot \sqrt{\varepsilon_{r, e f f}} \cdot l} .
$$

Die effektive Permittivität des unbelasteten Tastkopfs kann damit direkt ermittelt werden Mit diesem Ergebnis kann dann durch Einsetzen in (1) die effektive Permittivität für beliebige Belastungen des Tastkopfes bestimmt werden. Abb. 5 zeigt den Vergleich von theoretisch berechneten Realteil der Permittivität von Wasser-Ethanol-Gemischen unterschiedlicher Konzentration nach [13] und gemessenen Realteil der Permittivität bei einer Kalibrierung mit Luft und DI-Wasser. Es ist eine gute Übereinstimmung zu erkennen. Abweichungen können neben Messfehlern und Ungenauigkeiten bei der Herstellung der
Gemische, vor allem durch die starke Temperaturabhängigkeit der Permittivität von Wasser und Ethanol auftreten. Während mit dieser Kalibrierung gute Ergebnisse für den Realteil der Permittivität erzielt werden, ergeben sich sehr große Abweichungen beim Vergleich zwischen theoretisch berechneten Imaginärteil der Permittivität der WasserEthanol-Gemische und gemessenem Imaginärteil der Permittivität. Daher wird im Folgenden mit Ethanol und DI-Wasser kalibriert, da Ethanol mit einer Permittivität von $\varepsilon_{\text {Ethanol }}=24-j 10\left(25^{\circ} \mathrm{C}\right)$ im Gegensatz zu Luft dielektrische Verluste aufweist, was sich positiv auf die Genauigkeit bei der Kalibrierung für den Imaginärteil auswirken könnte.

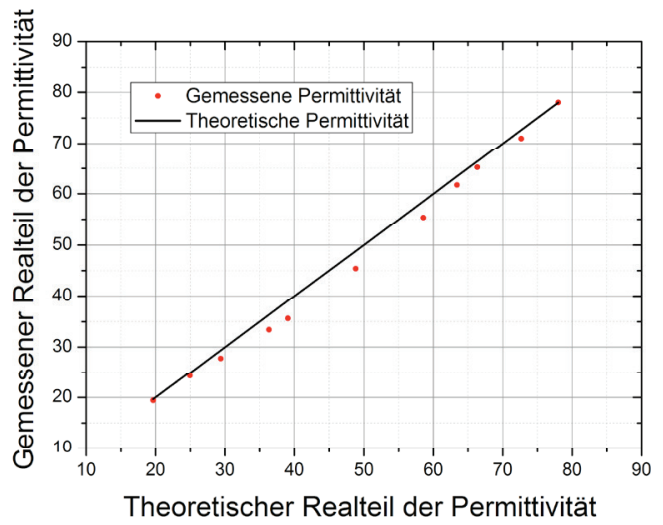

Abb. 5: Kalibrierung mit Luft und DI-Wasser: Vergleich von theoretischem und gemessenem Realteil der Permittivität von Wasser-EthanolMischungen.

In Abb. 5 ist der Vergleich vom theoretisch berechneten Realteil der Permittivität von Wasser-Ethanol-Gemischen unterschiedlicher Konzentration mit dem gemessenen Realteil der Permittivität bei einer Kalibrierung mit Ethanol und DI-Wasser dargestellt. Wie auch bei der Kalibrierung mit Luft und DI-Wasser ergibt sich eine gute Übereinstimmung. Es ist also möglich über einen großen Permittivitätsbereich von $\varepsilon_{r}^{\prime}=20$ bis $\varepsilon_{r}^{\prime}=80$ die relative Permittivität einer Probe anhand einer Messung der effektiven Permittivität mit dem Tastkopf zu bestimmen. In Abb. 7 ist der Vergleich vom theoretisch berechneten Imaginärteil der Permittivität von WasserEthanol-Gemischen unterschiedlicher Konzentration mit dem gemessenen Imaginärteil der Permittivität dargestellt. Im Gegensatz zur Kalibrierung mit Luft ist auch hier eine gute Übereinstimmung zwischen gemessenem und theoretischem Imaginärteil der Permittivität der Probe zu erkennen. Es können also die dielektrischen Verluste einer 
Probe im Bereich von $\varepsilon^{\prime \prime}{ }_{r}=2$ bis $\varepsilon^{\prime \prime}{ }_{r}=9$ mit dem Tastkopf genau bestimmt werden.

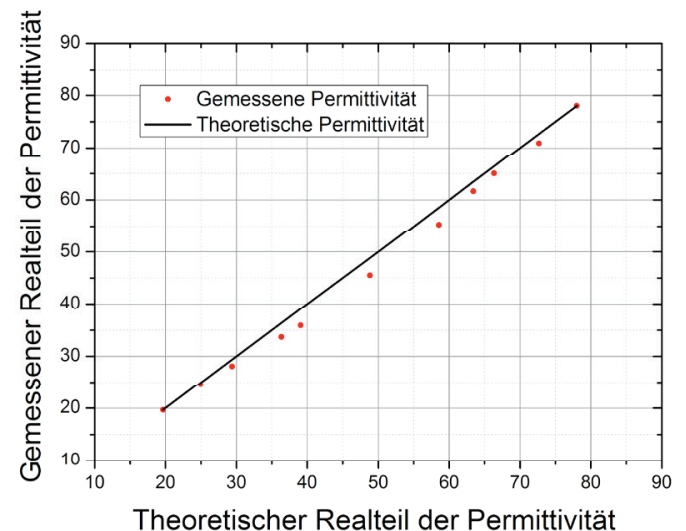

Abb. 6: Kalibrierung mit Ethanol und DI-Wasser: Vergleich von theoretischem und gemessenem Realteil der Permittivität von Wasser-EthanolMischungen bei $25^{\circ} \mathrm{C}$

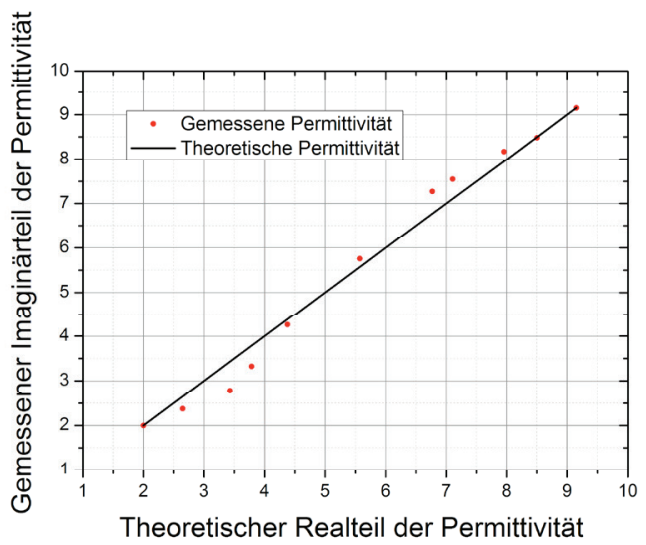

Abb. 7: Kalibrierung mit Ethanol und DI-Wasser: Vergleich von theoretischem und gemessenem Imaginärteil der Permittivität von Wasser-EthanolMischungen bei $25^{\circ} \mathrm{C}$.

\section{Zusammenfassung}

In dieser Arbeit wurde die Kalibrierung eines koplanaren Tastkopfes vorgestellt, mit der es möglich ist aus der gemessenen effektiven Permittivität die relative Permittivität einer Probe zu bestimmen. Dazu wurde eine Kalibrierung mit zwei Materialien mit bekannter Permittivität verwendet. Ein Vergleich zwischen Luft und DI-Wasser mit Ethanol und DI-Wasser als Kalibriermaterialien zeigt, dass in beiden Fällen gute Ergebnisse für die Bestimmung des Realteils der Permittivität der Probe erzielt werden können, allerdings bei der Bestimmung des Imaginärteils eine Kalibrierung mit Ethanol und DI-Wasser deutlich bessere Ergebnisse erzielt. Hiermit ist es nun möglich dielektrische Messungen über einen großen Permittivitäts- bereich mit dem koplanaren Tastkopf durchzuführen, womit die Anwendungsmöglichkeiten deutlich erweitert werden

\section{Referenzen}

[1] E. Nyfors, "Industrial Microwave Sensors - A Review", Subsurface Sensing Technologies and Applications, vol 1, pp 23-43, 2000

[2] J.L. Damez and S. Clerjon "Quantifying and predicting meat and meat products quality attributes using electromagnetic waves: An overview". Meat Science, vol. 95, pp 879896, 2013.

[3] Kent, M. "Hand-held instrument for fat/water determination in whole fish". Food Control vol. 1, pp. $47-53,1990$.

[4] B. Jian-Zhong, L. Shin-Tsu, W.D. Hurt: "Complex dielectric measurements and analysis of brain tissues in the radio and microwave frequencies", IEEE Transactions on Microwave Theory and Techniques 1997; 45(10):1730-1741.

[5] D. Popovic; M. Okoniewski: "Precision open-ended coaxial probe for dielectric spectroscopy of breast tissue". IEEE Antennas and Propagation Society International Symposium, 2002; 1:815-818.

[6] B. L. McLaughlin; P. A. Robertson: "Miniature open-ended coaxial probes for dielectric spectroscopy applications", J. Phys. D: Appl. Phys. 2007; 40:45-53.

[7] Clarke, B., Gregory, A., Cannell, D., Patrick M., Wylie, S., Youngs, I., Hill, G. (2003) A Guide to the Characterisation of Dielectric Materials at RF and Microwave Frequencies. London: National Physical Laboratory, Institute of Measurement and Control

[8] T. Reinecke, L. Hagemeier, S. Ahrens, Y. Doroshenko, M. Klintschar and S. Zimmermann (2015): A novel coplanar probe design for fast scanning of edema in human brain tissue via dielectric measurements, Sensors and Actuators B, vol. 220, pp. 522-527, 2015

[9] T. Reinecke, P. Biechele, V. Schulte, T. Scheper and S. Zimmermann: "Low-cost Sensor System for Non-Invasive Monitoring of Cell Growth in Disposable Bioreactors", Eurosensors XXIX, Procedia Engineering, vol. 120, pp. 548-551, Freiburg, Germany, 2015

[10] Baker-Jarvis, J.; Vanzura, E.J.; Kissick, W.A., "Improved technique for determining complex permittivity with the

transmission/reflection method," Microwave Theory and Techniques, IEEE Transactions on , vol.38, no.8, pp.1096,1103, 1990

[11] Meissner, T.; Wentz, F.J., "The complex dielectric constant of pure and sea water from microwave satellite observations," Geoscience and Remote Sensing, IEEE Transactions on , vol.42, no.9, pp.1836,1849, 2004

[12] J. Roelvink, S. Trabelsi, "A calibration technique for measuring the complex permittivity of materials with planar transmission lines" I2MTC: IEEE Int. Instrum. Meas. Technol. Conf. pp 1445-8, 2013 
[13] A. P. Gregory, and R. N. Clarke, "Tables of the complex permittivity of dielectric

reference liquids at frequencies up to 5 GHz," National Physical Laboratory Report MAT 23, 2009 